\title{
102 - Dementia and primary care - lessons from Europe: Symposium
}

Primary care doctors /General Practitioners are sometimes criticised for failing to recognise dementia in its early stages. Old age psychiatrists, neurologists or geriatricians take on the tasks of recognition, diagnosis (subtyping) and post-diagnostic support, and some Primary care doctors /General Practitioners welcome this division of labour as a way of avoiding the demanding tasks of dementia recognition and response. However, the rising prevalence of dementia syndromes in an ageing population is undermining the ability of secondary care specialists to fulfil their tasks of timely recognition, diagnosis and support. Primary care doctors /General Practitioners will be encouraged to take over some secondary care tasks. This will pose problems for Primary care doctors /General Practitioners, who may not have been trained to work with people with dementia, and who may not be able to incorporate such work into their practice even when trained. This symposium will explore the current and future challenges Primary care doctors /General Practitioners face in recognising and responding to dementia, and outline some lessons from four European countries (Ireland, Spain, Portugal and England). Themes to be presented include:

- Barriers to the recognition of dementia,

- The dementia-specific educational needs of Primary care doctors/General Practitioners,

- Interprofessional education of community-based primary care teams,

- Case management of people with dementia in primary care,

- IT solutions to problems of support for people with dementia.

- Effective dementia-care interventions in primary care post-diagnostic care pathways,

- Systemically-inspired brief interventions in primary care.

Chair: Emeritus Professor Steve lliffe, Centre for Ageing Population Studies, University College London [England]; co-chair: Professor Manuel Gonçalves-Pereira, Professor of Behavioural Medicine and Psychiatry, Nova Medical School, Universidade Nova de Lisboa [Portugal]

Speakers:

Dr Tony Foley, Lecturer Department of General Practice, University College Cork [Ireland]

Professor Manuel A. Franco, Head of Psychiatry and Mental Health Department.

University Rio Hortega Hospital (Valladolid) [Spain]

Dr Conceição Balsinha, General Practitioner \& Assistant professor/PhD student, Nova Medical School, Universidade Nova de Lisboa \& Professor Manuel Gonçalves-Pereira, Nova Medical School, Universidade Nova de Lisboa [Portugal]

Emeritus Professor Steve Iliffe, Centre for Ageing Population studies, University College London [England]

\section{3 - Risk and prevention of dementia: from observation to implementation}

Sebastian Köhler $^{1}$ (chair), Kay Deckers ${ }^{1}$, Edo Richard ${ }^{2,3}$, Rejko Krüger ${ }^{4,5,6}$

${ }^{1}$ Alzheimer Centrum Limburg, School for Mental Health and Neuroscience, Department of Psychiatry and Neuropsychology, Maastricht University, Maastricht, The Netherlands 